\title{
Fatigue of Intermittently Stimulated Quadriceps during Imposed Cyclical Lower Leg Movements
}

\author{
Henry M. Franken, Student Member, IEEE, Peter H. Veltink, Member, IEEE, \\ Marc Fidder and Herman B.K. Boom, Member, IEEE. \\ University of Twente, Department of Electrical Engineering, \\ P.O. Box 217, 7500 AE, The Netherlands.
}

\begin{abstract}
During prolonged experiments the influence of knee angular velocity, and stimulation parameters (interpulse interval (IPI), duty cycle (DC), number of pulses per cycle (NP)) on fatigue-induced torque decline of paralyzed human quadriceps was studied. Identification of torque-angle and -angular velocity was also performed. The overall loss of maximum torque (MT) and torque-time integral (TTI) per cycle during sustained intermittent stimulation during isokinetic movement had a typical exponential decay reaching asymptotic values. Larger knee velocities resulted in a significantly faster and relative larger decay of MT and TTI. The rate and relative magnitude of fatigue during concentric contractions are in direct relation to NP. The results may be valuable in the design of optimal control systems for FES which pursue minimization of muscle fatigue.
\end{abstract}

\section{INTRODUCTION}

A major issue in the control of functional electrical stimulation (FES) of paralysed muscles is the decay of muscle force as a result of fatigue under sustained (continuous and intermittent) stimulation [1-3, (2: in press)]. Stimulated paralyzed human quadriceps fatigue under isometric condition can be described by an exponential decay $[2,3]$. In this study the torque of intermittently stimulated paralysed human knee extensors during (isokinetic) cyclical lower leg movements has been investigated.

\section{METHODS}

A. Subjects

The subjects who participated in this study were complete T5-T6 level spinal cord injured patients. Al had normal excitable quadriceps muscle and had been enrolled in the FES training program of the rehabilitation center 't Roessingh (Enschede, The Netherlands).

\section{B. Protocol}

A protocol of 7 fatigue trials (FT) was designed to compare overall loss of tetanic torque at the knee joint during sustained intermittent stimulation at different isokinetic velocities. The angle and velocity ranges were limited by the anatomical restrictions of the lower leg, the restrictions imposed by the dynamometer bench and the desire to maintain a constant cycle time (2 s.), comparable to a walking cycle. The experimental set-up is shown in fig. 1. The influence of IPI (20 and $40 \mathrm{~ms})$, DC ([ontime/cycle time]: 16 and $32 \%$ ) and NP ([ontime/IPI]: 16 and 32), at isokinetic joint movement, was investigated. Pulsewidth $(\mathrm{PW}=300 \mu \mathrm{s})$ and stimulation amplitude (SA $=100 \mathrm{~mA}$ ) were set to obtain maximal recruitment. Three muscle identification trials, defermining the torque-angle (isometric measurement) and torque-angular velocity (isokinetically measured at $50 \mathrm{deg}$.) relations, were performed. Force, knee angular position, and velocity were sampled at $100 \mathrm{~Hz}$. These signals and stimulus data were stored on disk for off-line analysis.

\section{Off-line data analysis}

From the active torque, the maximum torque (MT) and torque-time integral per swing (TTI) are calculated. "Fatigue parameters", describing the decay of the MT and TTI as a function of time (one sample per cycle), were derived by curve-fitting the following equation to the data:

$$
\xi(t)=\xi_{\max } \cdot\left[\left(1-\xi_{\mathrm{o}}\right) \cdot \exp \left(-t / \tau_{\xi}\right)+\xi_{\mathrm{o}}\right]
$$

where $\xi$ is either MT or TTI, $\mathrm{t}$ is time, $\xi_{\max }$ is the maximum achieved in the considered trial, ${ }^{\top} \xi$ is the time constant of decay in $\xi$ due to fatigue, and $\xi_{0}$ is the relative asymptotic value to which $\xi(\mathrm{t})$ descends. The first part of the data (approximately 10 samples) which increases to $\xi_{\max }$ has been ignored. Equation (1) is fitted iteratively by minimizing the resulting RMS deviation from the experimental data (see also [2]).

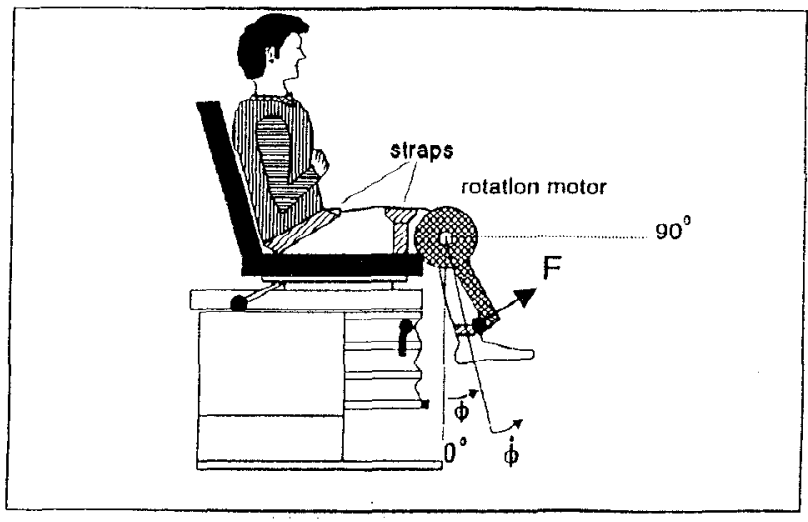

Fig. 1. Schematic of experimental set-up. The angular position and velocity, measured at the motor axis, are defined positive in extension motion with zero as indicated. The patient is strapped at the hip and knee to measure knee torque (at the tibia) only and to ensure static position of the body. 


\section{RESULTS}

A. Measured and estimated signals

Fig. 2 displays a typical registration of measured and estimated signals for one cycle.
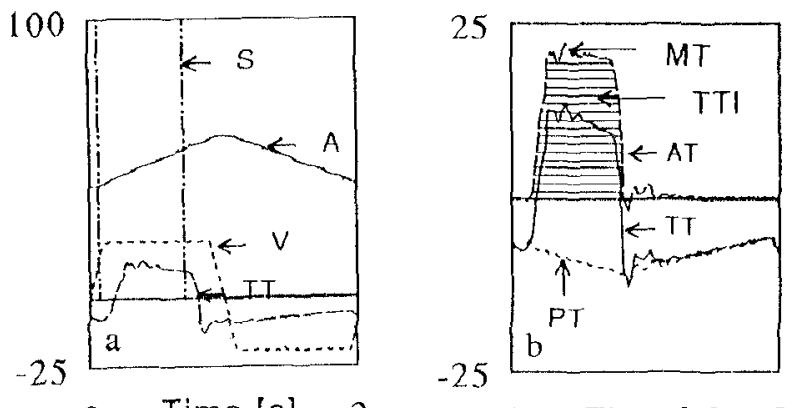

0 Time [s] 2
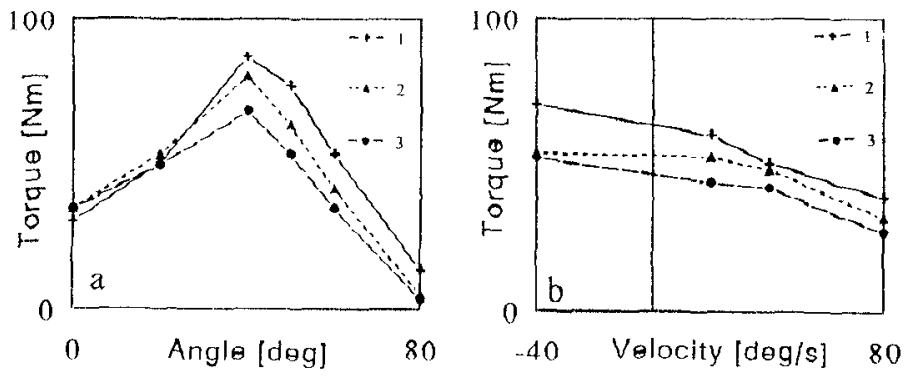

Fig. 4. Identification results of $T N$. (1): prior to FT 1; (2): after FT 3, (3): after FT 7. (IPI = $20 \mathrm{~ms})$ a: Torque-angle relation. b: Torque-angular velocity relation.

\section{DISCUSSION}

The apparent dependence of the quadriceps fatigue curve on the isokinetic knee joint velocity has not been reported before. The dependence might be explained by lower metabolic efficiency (shortage in ATP or high IMP concentrations) of the total muscle or by complete exhaustion of fast twitch fibers, which are more susceptible to FES than slow twitch fibers.
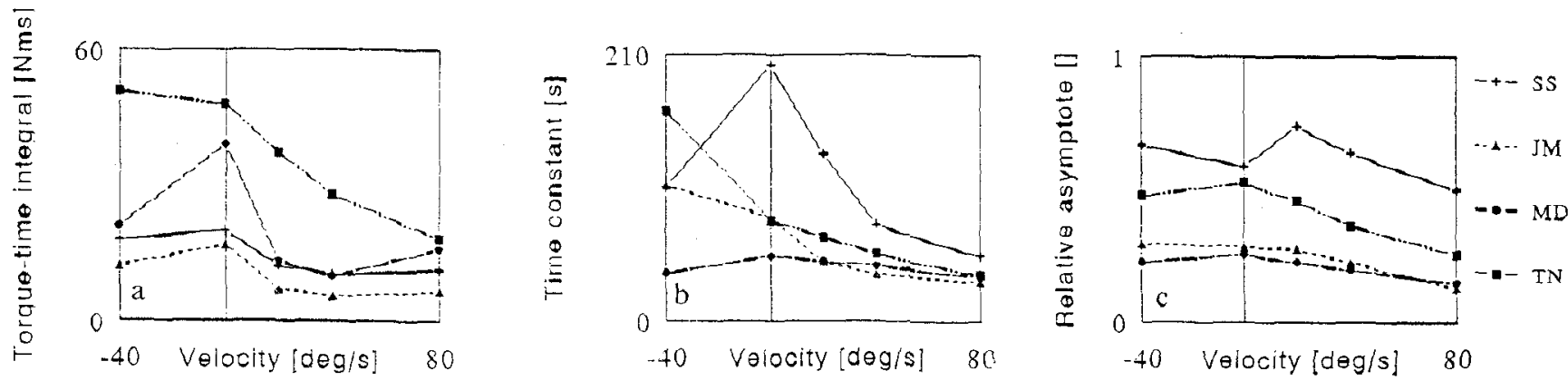

Fig. 3. Different velocities refer to seperate fatigue trials with identical stimulation parameters. (Subjects: $S S, J M, M D, T N$ ) a: $T T l_{\text {max, }}$ b: $\tau_{\text {Tir }}$ c: $T T I_{0}$

B. Fatigue parameters

Fig. 3 depicts the fatigue parameters estimated from the decay in TTI. TTImax displays a Hill type dependence on velocity, indicating comparable muscle condition for each trial. The graphs for $\tau_{\text {TII }}$ and TTIo show that higher velocities result in a significantly larger and faster decay of TTI $(\alpha<0.05)$. Variations in stimulation parameters indicated that the rate and magnitude of fatigue for concentric contractions are in direct relation to NP. The fatigue parameters for MT are similar.

C. Muscle dynamics.

A typical Gaussian-type dependence of the isometrically generated torque on the angle was found (fig. 4a). The measured torque-angular velocity relations supports the Hill equation. The relations alter due to fatigue. The decline in torque output due to fatigue is larger at higher velocities (see also fig. $3 \mathrm{c}$ ).
Concerning stimulation parameters, the results indicate that, within the boundary conditions for a given task, NP should be minimized to postpone fatigue. This can be accomplished by minimization of DC and/or 1/IPI. The results may contribute to the derivation of an optimization criterion, describing muscle fatigue as a function of both joint movement and stimulation parameters.

\section{REFERENCES}

1. Andrews B.I, Barnett RW, Phillips GI and Kirkwood CA: Rule-hased control of a hybrid Fes orthosis for assisting paraplegic locomotion. Atumedica

A $A$ and Veltink PII: Fatigue during finite state control Juring neturomuscular stimulation, Progress it Brain Research, in press.

3. Levy M. Marahi $I$ and Susak $Z$ : Recritment, force and fatigue characteris lics of quadriceps muscles of puraplegics isometrically activated by

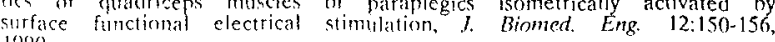

